\title{
QF-PCR AS A RAPID TECHNIQUE FOR ROUTINE PRENATAL DIAGNOSIS OF FETAL
} ANEUPLOIDIES

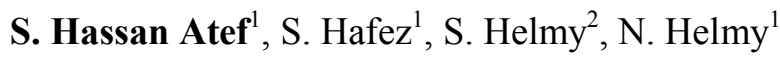 \\ ${ }^{l}$ Clinical Pathology, Ain Shams University, ${ }^{2}$ Prenatal Diagnosis and Fetal Medicine Department, National \\ Research Center, Cairo, Egypt
}

Background: The most common chromosomal abnormalities are aneuploidies of chromosome 21, 18, 13, X and Y. Prenatal diagnosis of fetal aneuploidies is routinely done by traditional cytogenetic culture, a major drawback of this technique is the long period required to reach a diagnosis. In this study we evaluated the QF-PCR as a rapid technique for prenatal diagnosis of common aneuploidies

Method: This work was carried out on Sixty amniotic fluid samples taken from patients with one or more of the following indications: Advanced maternal age (3 case), abnormal biochemical markers (6 cases), abnormal ultrasound (12 cases) or previous history of abnormal child (39 cases).Each sample was tested by QF-PCR and traditional cytogenetic. Aneuploidy screenings were performed amplifying four STRs on chromosomes 21, 18, 13, two pseudoautosomal,one X linked, as well as the AMXY and SRY; markers were distributed in two multiplex QFPCR assays (S1 and S2) in order to reduce the risk of sample mishandling .

Results: All the QF-PCR results were successful, while there was two culture failures, only one of them was repeated. No discrepancy was seen between the results of both techniques. Fifty six samples showed normal patterns, three sample showed trisomy 21 , successfully detected by both techniques and one sample showed normal pattern by QF-PCR but could not be compared to the cytogenetics due to culture failure, the pregnancy outcome of this case was a normal baby.

Conclusion: Our study concluded that QF-PCR is a reliable technique for prenatal diagnosis of the common chromosomal aneuploidies. 